\title{
High correlation between absolute psychophysical threshold and the scotopic threshold response to the same stimulus
}

\author{
Stuart L Graham, Vaegan
}

\begin{abstract}
The scotopic threshold response (STR) is a negative potential to low intensity light recorded from the dark-adapted retina. It reflects inner retinal function. The STR is now recorded routinely as part of our electroretinography protocol. The projected absolute threshold calculated from the amplitude versus intensity function for the STR has been found to correlate well $(r=0.59)$ with the absolute subjective threshold to the same stimulus. The correlation holds for about $1.5 \mathrm{log}$ units above normal. With further elevation the STR is usually abnormal or absent as b wave threshold is approached. The correlation would have been much greater were it not for this truncated range over which both are recordable. This report includes our findings in 127 patients and examines several disease groups. When the STR had a reduced and abnormal intensity series or was absent, the subjective threshold was elevated in almost all cases $(92 \cdot 2 \%)$. The converse relationship also held. When there was a discrepancy, recording problems were usually identified. Since the STR requires difficult, time consuming signal averaging for reliable recording, it may be adequate to record the subjective threshold alone to provide a relatively easily recordable indicator of inner retinal function. Additional STR recording will seldom be warranted.
\end{abstract}

The scotopic threshold response (STR) has been shown in man to be a unique component of the normal electroretinogram (ERG). ${ }^{1-3}$ It is a negative potential in response to the onset of illumination of intensities $1 / 2-2$ LU above absolute threshold. It occurs below the b-wave threshold, is dependent on rods at dim illumination, and probably reflects inner retinal function. ${ }^{46}$ Its origin has been proposed as being amacrine cells, ${ }^{3}$ and it has been reported as abnormal in diseases affecting the inner retinal circulation such as diabetic retinopathy. ${ }^{6}$ Since it is rod driven, it is also lost in disorders such as retinitis pigmentosa (RP), though its behaviour in the early stages of the disease has yet to be defined.

We have noticed that a raised absolute subjective threshold (recorded to blue strobe flash at the depths of dark adaptation) is usually seen in conjunction with an abnormal STR recorded with the same coloured flashes. The projected absolute threshold, obtained by extrapolating from the STR intensity series, shows good correlation with the subjective threshold, and this relationship holds in all disease groups tested. The significance of this is that the subjec- tive absolute threshold is a relatively fast and reliable test to perform, in contrast with the STR threshold. Each STR is a small signal $(<15 \mu \mathrm{V})$, and several prolonged averages must be obtained at each intensity before the threshold can be estimated. The subjective absolute threshold may therefore be a simpler addition to the electroretinography protocol than the STR and provide further information on the integrity of inner retinal function, especially when combined with examination of other inner retinal parameters such as the oscillatory potentials (OPs) or pattern electroretinogram (PERG). ${ }^{7-10}$

\section{Material and methods}

We examined 127 patients from Prince of Wales Hospital Eye Clinic and from routine referrals to our electrophysiology unit. Before recording began the patients' pupils were dilated to at least $8 \mathrm{~mm}$ with tropicamide 1\% (Mydriacil) and phenylephrine hydrochloride $10 \%$ (Neosynephrine).

\section{SUBJECTIVE THRESHOLD RECORDING}

The subjective threshold of each eye was recorded after a minimum of 20 minutes of dark adaptation in response to a blue strobe flash. The flashes were presented every 5 seconds, and intensity was initially set at $-6 \cdot 3 \log$ units. One eye was patched while the other was tested. The patient was asked to count consecutive flashes. The flash intensity was raised or lowered according to the patient's response in $0.3 \mathrm{log}$ unit increments. This was sufficiently coarse for us to usually find a 'threshold' level, where detection was $100 \%$ and detection 0.3 LU dimmer was near $0 \%$. In a few uncertain cases a detection rate of $50 \%$ had to be taken as the threshold.

\section{STIMULUS PARAMETERS}

Subjects were positioned on a chin rest with their faces $2 \mathrm{~cm}$ from the centre of a $61 \times 61 \mathrm{~cm}$ milk plexiglass screen $5 \mathrm{~mm}$ thick. In control experiments (Vaegan, Arora, and Millar, unpublished observations) we verified the report of Arden et $a l^{11}$ that the screen in this position gave ERGs indistinguishable from those obtained with a ganzfeld. An industrial quality stroboscope (IEL, Melbourne, Model XD) with an 8 inch (20 $\mathrm{cm}$ ) parabolic reflector $15 \mathrm{~cm}$ from the other side of the screen delivered $10 \mu \mathrm{s}$ white flashes. Two filter wheels were set between the strobe and the screen so that one of three coloured filters (red, blue, or white) could be selected and the intensity could be attenuated in $0.3 \mathrm{LU}$ steps between 


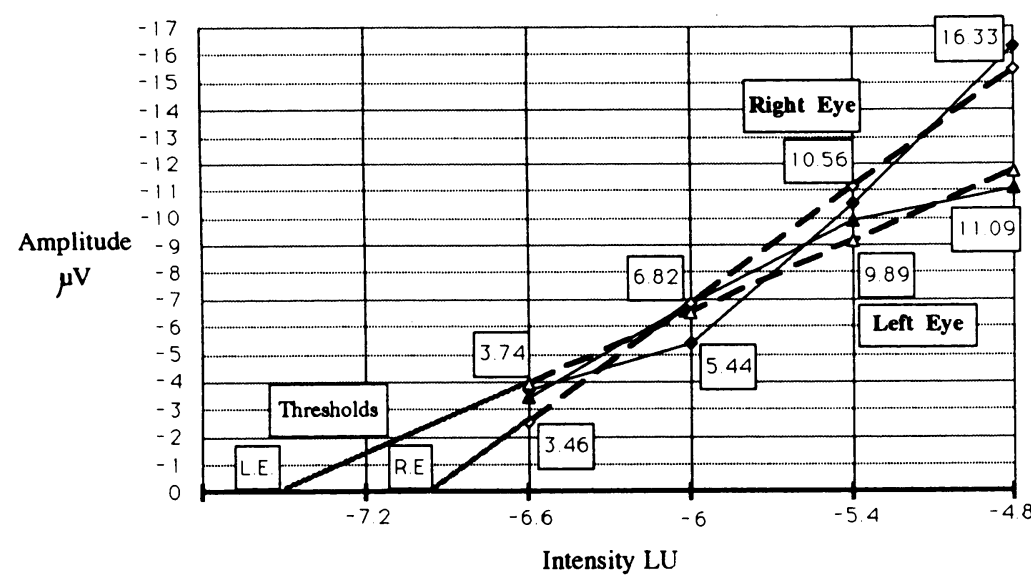

Figure 1 Example of plotting STR amplitudes and extrapolating an absolute threshold using a line of best fit. The values obtained for each patient were then compared with their subjective thresholds.
Table 1 Normal values for absolute subjective thresholds and for STR projected thresholds

\begin{tabular}{lll}
\hline Normals & STR & Subjective threshold \\
\hline No. of eyes & 28 & 30 \\
Mean age & $26 \cdot 8$ & $25 \cdot 5$ \\
Threshold & $-6 \cdot 93$, SD 0.38 & $-6 \cdot 87$, SD 0.09 \\
Gradient & $8 \cdot 9$, SD 3.0 & - \\
\hline
\end{tabular}

between here and 200 milliseconds. Cat STR peak times recorded by Arora, Vaegan, and Miller ${ }^{14}$ were used as a guide. It was essential to take special care with noise elimination to ensure reliable recording of small signals like the STR.

To eliminate mains noise, timing was driven by a counter, based on a mains signal zero crossing detector to give clock pulses at $2 \times$ mains frequency. The averager was triggered after an odd number of zero mains crossings, usually 67 , that is, 670 milliseconds, also 2670 to 4670 at higher intensities above b-wave semisaturation. The ongoing high frequency noise was never greater than $100 \mu \mathrm{V}$; if higher values were observed, electrodes were examined and corrected and the recording restarted.

Artefact rejection was carefully adjusted. In general this can be done in two ways: either varying gain to make a steady signal close to the limits of the artefact rejector or by varying its trigger limits. The first was not available to us because of our fixed gain amplitude. The second was readily accomplished, though with some loss in the number of useful bits in the 12 bit A to D converter. Limits could be dynamically adjusted during the test by setting a single parameter to ensure there were always a few rejections $(5 \%)$, however good the subject. System noise was \pm 30 to $\pm 50 \mu \mathrm{V}$. The tightest limit was $\pm 75 \mu \mathrm{V}$, while the most relaxed was never wider than $\pm 200 \mu \mathrm{V}$ in the STR range.

Continuous monitoring of the ongoing signal. Mains noise and artefacts which suddenly intruded were usually associated with the gold foil sticking to the tear wetted cheek after blinks. Electrodes were readjusted under red light to ensure that the bottom of the gold foil stood off the face (in accordance with Holder ${ }^{15}$ ). The experimenter and the subject were alerted to the presence of blinks or eye movements by computer beeps. The subject's task was to keep

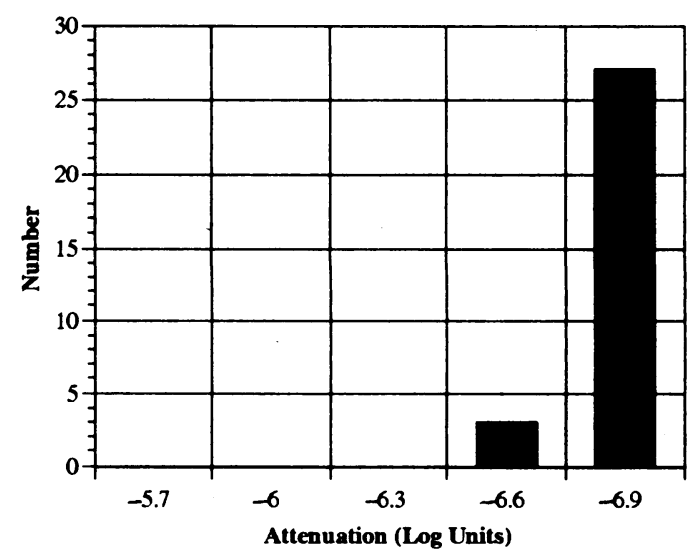

Figure 2 Absolute subjective thresholds for normal patients. Lower limit of testing was $-6.9 \mathrm{LU}$ and almost all patients achieved this level. $\mathbf{Q}=$ Number of eyes tested. To that the response had returned baseline after $384 \mathrm{~ms}$. Latency was chosen from values close to those published by Aylward, Vaegan, and Billson. ${ }^{2}$ Minima were chosen 
Table 2 Values for absolute subjective thresholds and for STR projected thresholds in different disease groups. The absent column includes those with a markedly abnormal STR intensity series such that a threshold could not be extrapolated. Note the large number of $R P$ cases and diabetics in this column

\begin{tabular}{|c|c|c|c|c|c|c|}
\hline \multirow[b]{2}{*}{ Disease group } & \multirow[b]{2}{*}{$\begin{array}{l}\text { No. } \\
\text { eyes }\end{array}$} & \multicolumn{3}{|c|}{$S T R$ - projected threshold } & \multicolumn{2}{|c|}{ Subjective threshold } \\
\hline & & Absent & $\begin{array}{l}\text { Mean } \\
(-L U)\end{array}$ & $S D$ & $\begin{array}{l}\text { Mean } \\
(-L U)\end{array}$ & $S D$ \\
\hline$R P$ and retinal dystrophies & 52 & 45 & 4.98 & 0.87 & $4 \cdot 81$ & 1.08 \\
\hline Cone dystrophy & 24 & 4 & $6 \cdot 39$ & 0.85 & 6.53 & 0.35 \\
\hline Diabetes & 36 & 18 & 6.03 & 0.69 & 5.65 & 0.87 \\
\hline Pre laser & 22 & 8 & 5.99 & 0.75 & $5 \cdot 43$ & 1.05 \\
\hline Post laser & 14 & 10 & $6 \cdot 10$ & 0.37 & 6.00 & 0.24 \\
\hline CRVOs & 4 & 4 & - & - & $6 \cdot 38$ & 0.29 \\
\hline \multicolumn{7}{|l|}{ Glaucoma and ocular } \\
\hline hypertension & 24 & 8 & $6 \cdot 62$ & 0.64 & $6 \cdot 27$ & 0.73 \\
\hline Optic nerve disease & 34 & 8 & $6 \cdot 50$ & 0.91 & $6 \cdot 20$ & 0.71 \\
\hline Macular disorders & 14 & 1 & 6.57 & 0.41 & 6.60 & 0.37 \\
\hline \multicolumn{7}{|l|}{ Stargardt's disease/flavimaculatus } \\
\hline and Best's disease & 16 & 6 & $6 \cdot 51$ & $0 \cdot 30$ & $6 \cdot 53$ & 0.48 \\
\hline Mixed disease & 20 & 8 & $6 \cdot 88$ & 0.65 & $5 \cdot 87$ & 0.85 \\
\hline
\end{tabular}

these beeps to a minimum, since each one was associated with a rejected sweep.

Mains shielding. The cables for active and indifferent were shielded by the earth lead to within $10 \mathrm{~cm}$ of the subject.

The STR amplitudes were plotted against intensity and a least squares estimated straight line was fitted to the monotonically increasing part of the curve. Because the line is fully

CASES WITH ABNORMAL STRS UNABLE TO PROJECT THRESHOLD
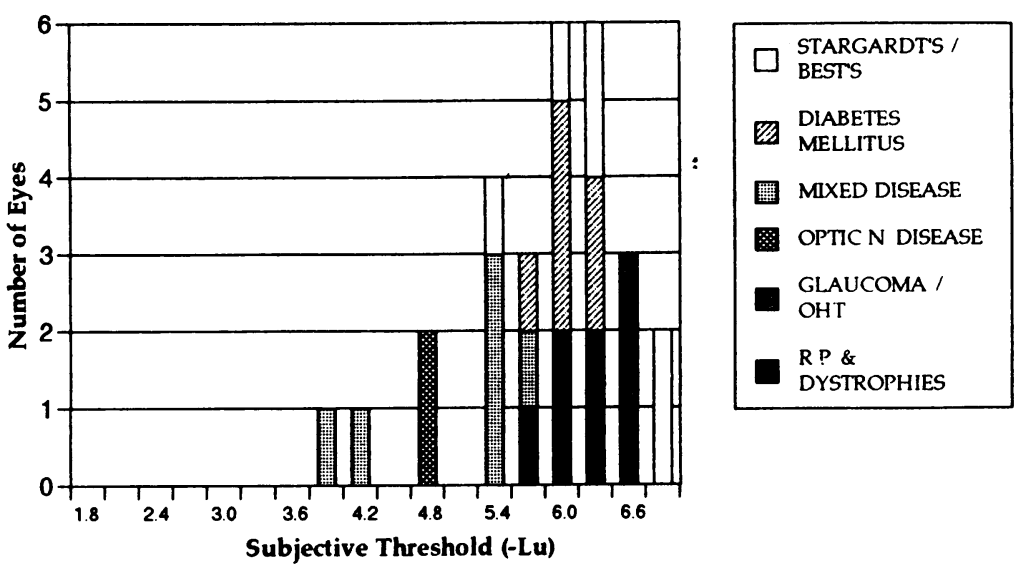

CASES WITH ABSENT STRS

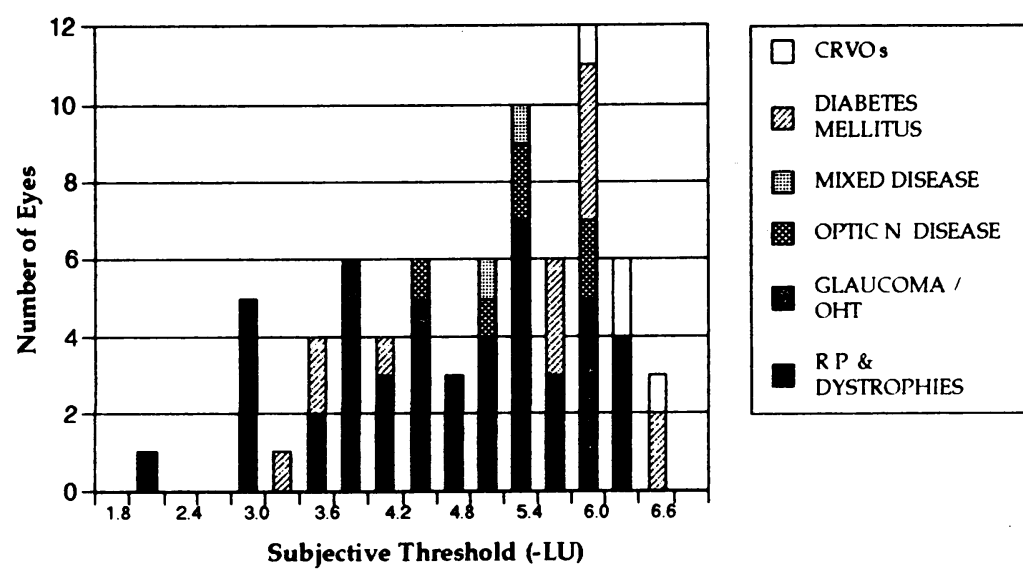

Figure 3 Charts showing the subjective thresholds in those patients in whom the STR was absent, or reduced such that an absolute threshold could not be determined. Of these patients $92 \%$ recorded a subjective threshold above or at $-6 \cdot 3 L U$ (which is elevated).

As expected the STR did not persist in most cases of $R P$, since it is rod dependent, although it was preserved in most of the cone dystrophies. The previously described finding of its reduction in diabetic retinopathy was observed. It was also absent in CRVO's. No definite pattern of reduction was identified in other disease groups (see Figure 6 also). described by two parameters - gradient (in $\mu \mathrm{V} /$ $\log$ unit) and threshold (the zero intercept) - a more complete assessment of the STR function is achieved in the form of a gradient of increasing response and an extrapolated threshold. Figure 1 shows an example using a normal patient.

Problems in recording the STR include: (1) The STR can be recorded only across a limited range of intensities (1.5-2 LU). (2) Variable $\mathrm{V}_{\max }$ and amplitude intensity function due to $\mathrm{b}$ wave intrusion. (3) Confusion with slow PIII (which occurs later and at slightly higher intensities). As a general rule we found that STR peak times were $220 \mathrm{~ms}$ at most and became earlier as the response grew larger, whereas slow PIII was always later than $240 \mathrm{~ms}$. When the STR was reduced with an elevated threshold and the bwave/PIII complex was normal, the STR and slow PIII could be confused. (4) High noise, including eye movements. (5) Problems of fitting lines to a limited series of imprecisely located points to obtain an accurate projected threshold.

\section{Results}

The normal values are shown for patients tested in our laboratory in Table 1 and their distribution in Figure 2. Almost all normal persons achieved a subjective threshold of $-6.9 \mathrm{LU}$, with a lesser number recording $-6.6 \mathrm{LU}$. This gave a mean of $-6.87 \mathrm{LU}$ (SD 0.09 LU). For the STR the mean projected threshold for our normals was -6.93 LU (SD $0.38 \mathrm{LU})$. The results, except for one case, represent recordings made on the same patient at a single recording session.

In Table 2 the results are included for the broad disease groups. Whereas a subjective threshold is always recordable (even if raised significantly), the STR intensity series is often abnormal in disease, and therefore this table includes threshold values only in those patients in whom they were recordable. Figure 3 shows the remaining patients in whom the STR was either abnormal or absent. In all but eight of these eyes the subjective threshold was $-6 \cdot 3 \mathrm{LU}$ or above, which is outside our range for normal persons. Therefore when the STR had either an abnormal intensity series or was absent, $92 \cdot 2 \%$ of eyes tested had an elevated subjective threshold.

The graphs shown in Figures 4 and 5 record the projected ERG thresholds derived from the STR series plotted against the subjective thresholds for each patient, Figure 4 is a summary of all patients where both an STR and a subjective threshold could be recorded. The calculated regression lines have been drawn in.

There is a significant correlation between the two recordings of 0.59 . This correlation would have been higher but for two limiting factors. Firstly, the lower limit of our equipment for subjective thresholds was $-6.9 \mathrm{LU}$, and clearly the majority of normal persons were at this level. Had we been able to test for lower levels some of these patients may well have had thresholds of $-7 \cdot 2 \mathrm{LU}$ or less. Secondly, at levels above $-5 \cdot 0$ LU, the b-wave begins to intrude on the STR, eventually cancelling it (see Figure 6 for example of normal patient). The patients with thresholds 


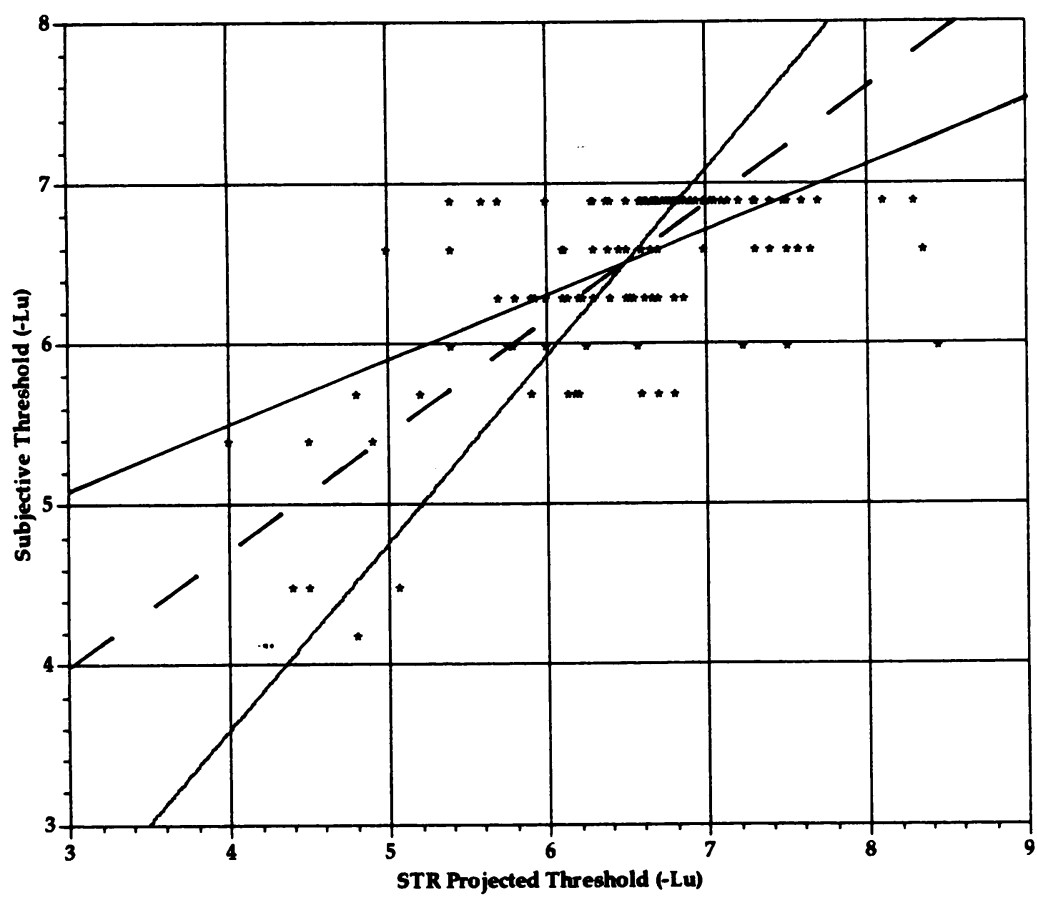

Figure 4 Summary of all patient groups plotting STR projected threshold against subjective threshold. Linear regression lines are included. Correlation $r=0.59$. Note the flattened distribution at $-6.9 \mathrm{LU}$ representing our lower limit of the subjective threshold recording. Also very few patients are seen to have a recordable STR above a threshold of $-5 \cdot 4 \mathrm{LU}$. These two factors have limited the correlation achieved.

elevated into the b-wave range would therefore have their STRs reduced or cancelled, and many of these patients may be seen in Figure 3. A further example of this could be in some RP patients when a delayed b-wave intrudes on the STR directly and cancels it earlier, though we have seen some RP patients in whom the b-wave threshold is also elevated and the STR may be reduced but preserved.
It can be seen from Figures 2, 3, and 4 that if the subjective threshold is above $-6.0 \mathrm{LU}$ there is a very high incidence of either an abnormal STR threshold, or of the STR being reduced in amplitude and range, or even absent. There were a few cases of artificially low STR thresholds $(<-8.0 \mathrm{LU})$; also eight eyes recorded a reduced or absent STR at subjective thresholds of $-6 \cdot 6$ LU or better. We believe, from examining the traces in these subjects, that they mainly represent STR recording errors with a combination of variable wave forms and incomplete or truncated intensity series, which makes extrapolating a threshold unreliable.

It is not the purpose of this paper to report the various changes of the STR seen in individual diseases. However, in general it can be seen that there were reductions in the STR threshold in diabetes as previously described by Aylward. ${ }^{\circ}$ There were more marked losses in those who had undergone laser treatment, as expected with large areas of retina ablated, but the correlation still held in these patients. There were significant reductions in most cases of RP, which is consistent with widespread rod losses. The STR was also absent in the four central retinal vein occlusions reflecting inner retinal ischaemia. It was preserved in the cone dystrophies.

\section{Discussion}

Our recordings show that, when the subjective threshold is raised, the STR threshold is also raised. This relationship holds for about $1.5 \mathrm{log}$ units, above which the STR is usually abnormal or absent. Conversely, when the STR is of reduced amplitude, abnormal form, or has a raised threshold, the subjective threshold is also abnormal In those few cases in which there was
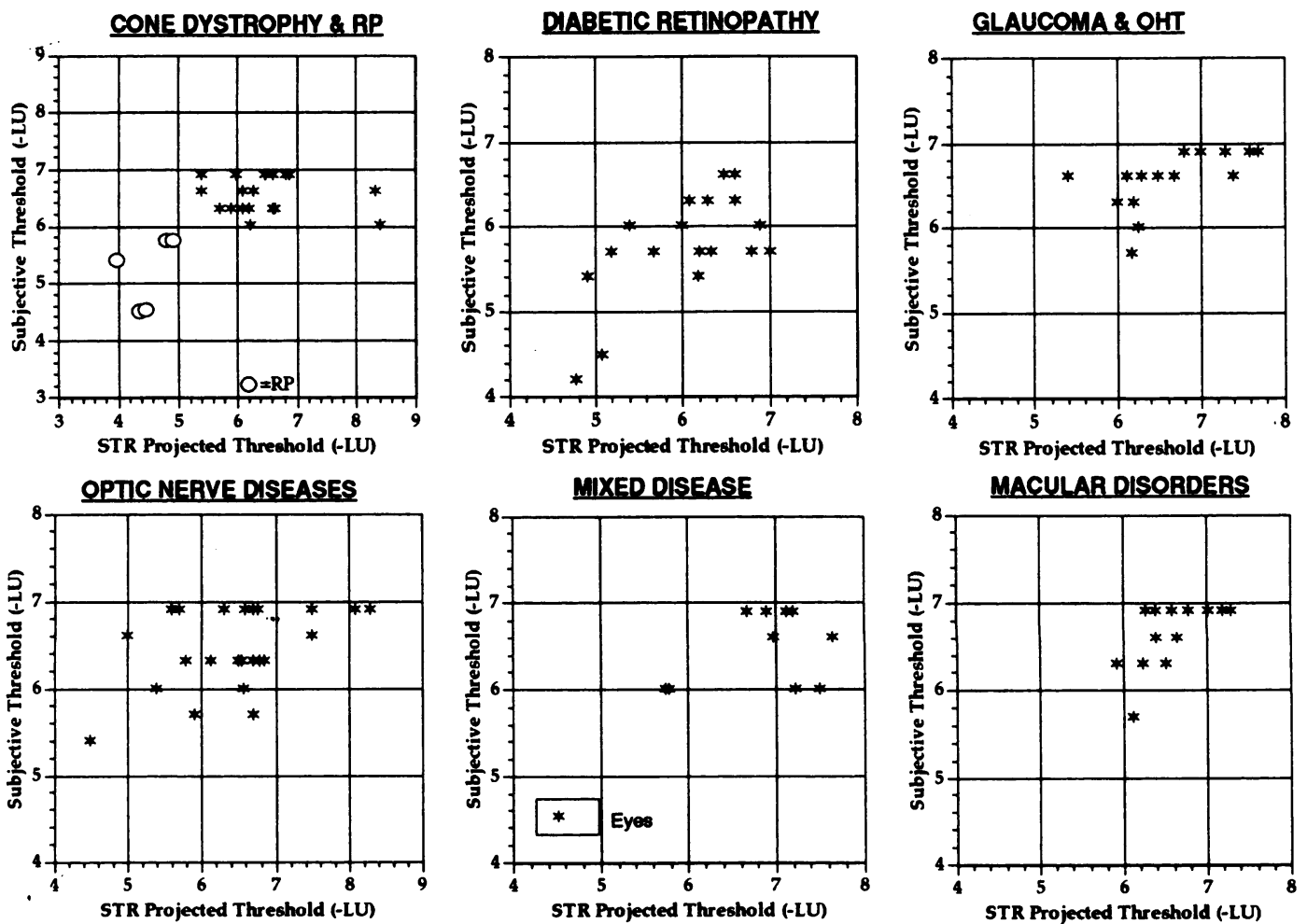

Figure 5 Individual patient groups showing STR projected threshold plotted against the subjective threshold. Correlation between the two methods seems to hold for different disease groups. The few RP patients who had an STR are shown as circles. 


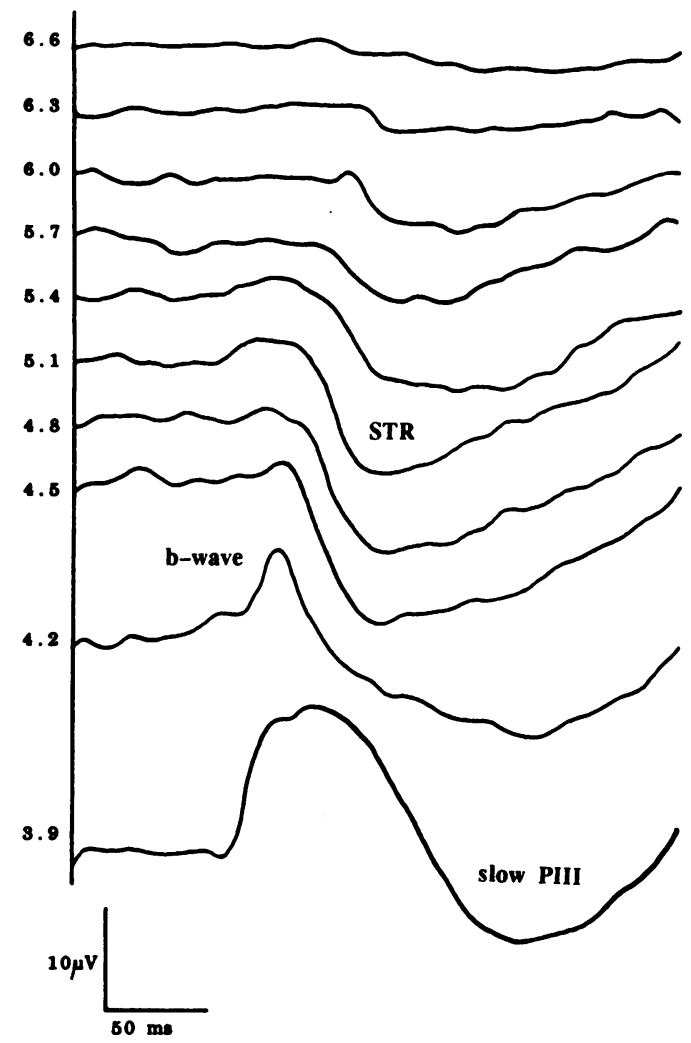

Figure 6 Example of normal STR intensity series. Note increasing amplitude of STR until b-wave threshold is reached. The STR becomes swamped by the b-wave by $-4 \cdot 2 L U$.

no agreement between the two responses it was usually the STR that was suspicious. We believe that this reflects recording difficulties with the STR rather than a true discrepancy between the responses.

It is clear that a raised subjective threshold is by no means a specific indicator for any one disease process, but shows subtle changes at these very low luminances which seem to reflect a disturbance of the integration of rod responses. This normally occurs in the process of spatial summation at the level of the amacrines, so it is not surprising that diseases that alter the STR (which is thought to originate at this level) will also effect the subjective threshold.
Since the amplitude of the STR is very small (8-20 $\mu \mathrm{V}$ ), recording is subject to frequent artefact intrusion and requires multiple sweeps to achieve a reliable trace. At least five traces taking over 15 minutes need to be recorded to ensure there are enough data. Recording the subjective threshold, however, is simple, and the only source of error here is patient confidence in reporting the visual stimulus at low levels. Thus the subjective threshold may be an easy alternative to the STR in co-operative patients and still provide additional information on both rod function - as has been its traditional role in conditions such as RP - and on the state of the inner retina in diabetic retinopathy or central retinal vein occlusion for example. If a questionable elevated subjective threshold is found, electrophysiological confirmation with an STR intensity series is still possible.

1 Sieving PA, Frishman LJ, Steinberg RH. Scotopic threshold response of proximal retina in cat. $\mathcal{F}$ Neurophysiol 1986; 56: 1049-61.

2 Aylward GW, Vaegan, Billson FA. The scotopic threshold response. Clin Vision Sci 1989; 4: 373-7.

3 Sieving PA, Nino C. Scotopic threshold response of the human electroretinogram. Invest Ophthalmol Vis Sci 1988; 29: 160814.

4 Wakabayashi K, Gieser J, Sieving PA. Aspartate separation of the scotopic threshold response from the photoreceptor awave of the cat and monkey. Invest Ophthalmol Vis Sci 1988; 29: 1615-22.

5 Frishman LJ, Steinberg RH. Light-evoked increases in $(\mathrm{K}+) \mathbf{0}$ in proximal portion of the dark-adapted cat retina. in Neurophysiol 1989; 61: $1233-43$.

6 Aylward GW. The STR in diabetic retinopathy. Eye 1989; 3: 626-37.

7 Coupland SG. A comparison of oscillatory potentials and pattern ERG measures in diabetic retinopathy. Doc Ophthalmol 1987; 66: 207-18.

8 Bresnick GH, Palta $M$. Oscillatory potential amplitudes: relation to severity of diabetic retinopathy. Arch Ophthalmol 1987; 105: 929-33.

9 Wachmeister L. Basic research and clinical aspects of the oscillatory potentials of the electroretinogram. Doc Ophthalmol 1987; 66: 187-94.

10 Arden GB, Vaegan, Hogg CR. Clinical and experimental evidence that the PERG is generated in more proximal retinal layers than the FERG. Ann NY Acad Sci 1982; 388: $580-601$.

11 Arden GB, Carter RM, Hogg CR, et al. A modified ERG technique and the results obtained in $\mathrm{X}$-linked retinitis pigmentosa. Br f Ophthalmol 1983; 67: 419-30.

12 International Standardisation Committee. Standard for clinical electroretinography. Arch Ophthalmol 1989; 107: 816-9.

13 Vaegan. An improved method of constructing pattern electroretinogram electrodes. Doc Ophthalmol Proc Ser 1984; 40: 287-93.

14 Arora A, Vaegan, Millar TJ. The effect of kainic acid (KA) and N-methyl-D aspartate (NMDA) on the electroretinogram and morphology of the cat retina. Neurosci Lett 1989; 34: S54.

15 Holder GE. Update: recording the pattern electroretinogram with the Arden gold foil electrode. F Electrophysiol Technol 1988; 14: 183-90. 